\title{
Lateral guidance control using information of preceding wheel pairs
}

\author{
Gustav Grether, Andreas Heckmann, and Gertjan Looye \\ Institute of System Dynamics and Control, German Aerospace Center (DLR), \\ Muenchner Strasse 20, 82234 Wessling, Germany \\ gustav.grether@dlr.de
}

\begin{abstract}
The proceeding enhancement of sensor technology, data processing and communication opens a broad field to improve the dynamics of railway vehicles by controlled systems targeting e.g. passenger comfort and wear reduction. In terms of an integrated control structure, information of leading bogies can be used for an advanced control of the trailing ones, like it is recently applied in tilting trains. Such an approach has not yet been investigated for the lateral guidance of driven independently rotating wheels (DIRW). To evaluate the potential of a control using preview information, an integrated control structure is introduced in this work. The control is based on the concept of feedback linearization and considers characteristics of track trajectory and irregularity, which are obtained at a leading wheel pair. The control performance is optimized with the help of software-in-the-loop simulations and the results show a significant improvement of the running dynamics.
\end{abstract}

Keywords: lateral guidance, control, feedback linearization

\section{Introduction}

In the DLR project 'Next Generation Train' (NGT) a future high speed train concept is developed targeting vehicle speeds of $400 \mathrm{~km} / \mathrm{h}$, higher passenger comfort, energy efficiency and a significant wear reduction. Prospectively, the proceeding enhancement of sensor technology, data processing and communication will lead to a better knowledge of track and vehicle states in operation. Hence, controlled systems are investigated, processing the information to achieve the project goals of NGT. Consequently, the novel mechatronic running gear includes active primary and secondary suspension and an active guidance control. The intermediate wagons are equipped with two running gears, each with one DIRW. The wheels are mounted on the axle beam, which enables steering by a rotational degree of freedom around the vertical axis. The motors at each wheel serve as well for braking, traction and as actuators for the guidance control. Applying a torque in opposite direction at the left and the right wheel, the guidance control guaranties stable running dynamics of the NGT. Kurzeck [10] investigates the running dynamics of the NGT in multibody simulations and shows that the targeted wear reduction can be attained. The design and 
verification of a robust control for a 1:5 scaled roller rig is given in [5] and the control is transferred successfully to full scale multibody models of the NGT [4]. Moreover, information of leading bogies can be used for an advanced control of the trailing ones, like it is recently applied in tilting trains [7]. Supplying the information forehead, the time gap between the running gears can be used for data processing or a compensation of the vehicle inertia by the control. Although the guidance control for DIRW is as well in focus of other research projects [1], the possibility to use information of leading bogies is not investigated yet. Therefore, the potential of such an integrated control structure is evaluated in this work. The lateral guidance control is achieved with the concept of feedback linearization, enabling to explicitly address nonlinearities like the rail wheel contact in the control design. The control is extended to account for the major influences due to track trajectory and irregularities. In the following the control design and the underlying analytical model is explained. Results of multibody simulations are presented, showing the advantages of the integrated control for the lateral dynamics.

\section{Guidance Control}

\subsection{Analytical Model}

The analytical model described in [2] consists of three bodies. The left and right wheel are mounted on an axle beam, which is fixed to the inertial track system with a yaw spring damper. Extending the model by the nonlinear wheel rail profile leads to a nonlinear system $\dot{\mathbf{x}}=\mathbf{f}(\mathbf{x}, u)$ with the state $\mathbf{x}=\left(y_{w}, \dot{y}_{w}, \psi, \dot{\psi}\right.$, $\left.\omega_{r i}, \omega_{l e}\right)$. The lateral displacement of the axle beam regarding the track center is described with $y_{w} . \psi$ denotes the yaw angle of the axle beam and $\omega_{r i / l e}$ the wheel rates. To attain steering, the input control torque $u=\Delta \tau$ is applied in opposite directions at each wheel. The creep forces in the contact are calculated according to the linear Kalker law with constant coefficients [9]. Ideally, the carbody stays on the ideal track path and the wheel pair follows the disturbed track center in lateral direction to avoid flange contact. Hereby the secondary suspension decouples the motion of the carbody from the guidance dynamics of the running gear, so that the carbody mass can be neglected in lateral direction. However, for the normal contact force $Q_{N}$ the vertical load of the carbody is taken into account. The major nonlinearity of the system originates from the nonlinear rail wheel profile, visualized in Fig. 1. Most important for the system dynamics, is the rolling radius $r_{r i / l e}$, which influences the creepages and therefore the contact forces. The second important value is the contact angle $\delta_{r i / l e}$ effecting the direction of contact forces. Both values depend particularly on $y_{w}$. This relation is extracted from Simpack as displayed in Fig. 1 and approximated in the relevant area $y_{w}=[-10 \mathrm{~mm}, 7 \mathrm{~mm}]$ with exponential functions and the coefficients $r_{1-3}$ and $\delta_{1-4}$ :

$$
\begin{aligned}
& \delta_{r i / l e}\left(y_{w}\right)=\delta_{1} e^{ \pm \delta_{2} y_{w}}+\delta_{3} e^{ \pm \delta_{4} y_{w}} \\
& r_{r i / l e}\left(y_{w}\right)=r_{1}+r_{2} e^{ \pm r_{3} y_{w}}
\end{aligned}
$$



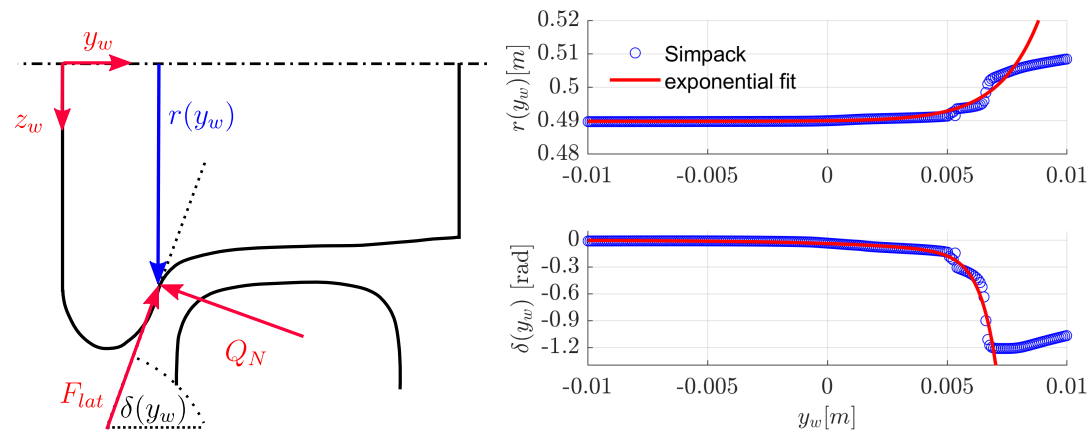

Fig. 1. Nonlinear rail wheel profile (le) and approximation of rolling radius and contact angle (ri)

Further simplifications are introduced to deduce a model suitable for the control design. In the lateral direction the motion is dominated from the lateral creep force $F_{\text {lat }}$ caused by spin and lateral creepage and the lateral amount of $Q_{N r i / l e}$. Hence, the longitudinal creep force is neglected and for the spin creepage only the main amount induced by the nominal wheel rate $\omega_{r i / l e} \approx \omega_{0}=-v / r_{0}$ is considered. Regarding the most common case of low traction requirements, the different wheel speeds allow for operations with minor longitudinal creepage despite of lateral displacements $y_{w} \neq 0$. Therefore, ideal rolling is assumed and a kinematic coupling exists: $\omega_{r i / l e}=\left(\dot{\psi}\left( \pm b-y_{w}\right)-v\right) / r_{r i / l e}$. The relation between wheel and yaw rate is dominated by the track gauge $b$ and the rolling radii. This way, the system state is reduced to $\mathbf{x}=\left(y_{w}, \dot{y}_{w}, \psi, \dot{\psi}\right)^{T}$ and consists of two equations for lateral and yaw motion.

\subsection{Control Design}

The lateral control design is dedicated to guaranty lateral stability in presence of disturbances, especially the track irregularities. The control has to face changing velocities, loads and track trajectories, which is challenging because of the highly nonlinear system. For these reasons, the concept of feedback linearization [6] is applied. This way, the nonlinearities are included in the model based control design and gain scheduling between linear designs in various operating points is avoided. Using the modeling language Modelica, the controller can be derived conveniently due to the automatic model inversion [11].

The deduced analytical model of the DIRW enables the model inversion in the cascaded the control structure displayed in Fig. 2. According to the concept of feedback linearization $\mathbf{x}$ has to be known for the feedback and the input in the two inverted equations of motion. Prospectively, the signals can be attained 
from observes in reality [3]. In this work, $\mathbf{x}$ is considered ideally. A peculiarity of the cascaded structure is the calculation of a desired yaw motion $\psi^{*}$ and $\dot{\psi}^{*}$, physically corresponding to a steering angle required for the desired lateral motion. Since no instable zero dynamics occur, an asymptotically stable trajectory following for a desired lateral position $y_{w}^{*}$ is established with the four controller gains $k_{i}$. Finally, the control is transferred via a functional mock up unit to a multibody model in Simpack. Therein, the controller performance is optimized with the DLR in-house optimization tool MOPS [8], considering lateral position and control effort on tracks with irregularities.

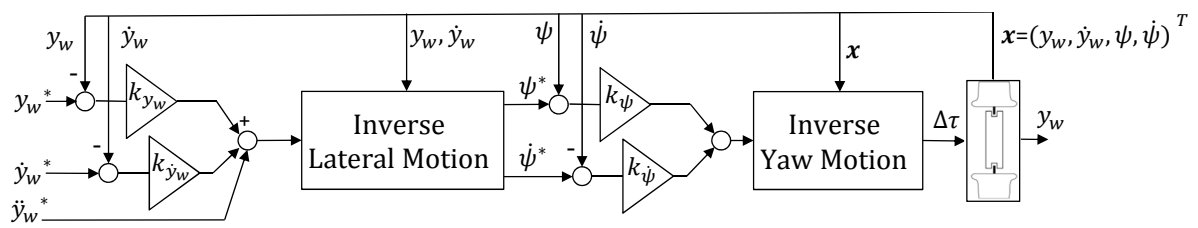

Fig. 2. Cascaded lateral control structure

\section{$3 \quad$ Extended Control}

\subsection{Influences}

The major influences on the lateral control arise from the track characteristic and are listed in Table 1. Firstly, these are the lateral track irregularities $y_{T}$. Secondly, lateral accelerations $a_{\text {lat }}$ occur in unbalanced curves, determined by the gravity $g$, superelevation angle $\varphi_{T}$, longitudinal velocity $v$ and curve radius $r_{T}$. Beyond that, gyroscopic effects $M_{G}$ arise in transition curves with $\dot{\varphi}_{T}$ and act directly on the yaw rotation $\psi$. The effect increases with the nominal wheel rate $\omega_{0}$ and the wheel Inertia $J_{y W h}$. Especially for the desired high speed this influences the steering enormously [4].

Table 1. Main Influences on lateral control

\begin{tabular}{|l|c|c|c|}
\hline \multicolumn{2}{|c|}{ Influences } & Dynamic & Approach \\
\hline curving & $a_{l a t}=g \varphi_{T}-v^{2} / r_{T}$ & slow & model input \\
\hline transition curve & $M_{G}=2 \omega_{0} J_{y W h} \dot{\varphi}_{T}$ & medium & model input \\
\hline lateral track irregularity & $y_{T}, \dot{y}_{T}, \ddot{y}_{T}$ & fast & desired trajectory \\
\hline
\end{tabular}

The frequency of the track irregularities depends mostly on the longitudinal velocity. Focusing on high speed trains, they occur with high frequencies. In 
contrast, the influences of the track trajectory $r_{T}, \varphi_{T}$, and $\dot{\varphi}_{T}$ change with a substantially lower dynamic. The introduced control design allows to feed variable model parameters with a slow dynamic directly into the inverse model without regards to the stability. Additionally, a desired lateral trajectory $y_{w}^{*}$ can be specified to account for the track characteristics. The required signals for the preview information are obtained at the leading bogies and pre-processsed with low pass filters and time delays. The cut-off frequencies of the filters and the time delays are optimized with MOPS in multibody simulations. The different approaches for the influences are visualized in Fig. 3 and explained in the following chapters.

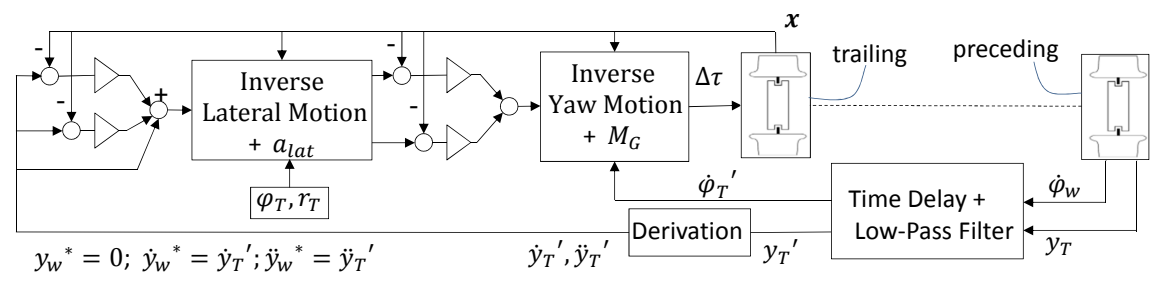

Fig. 3. Nonlinear control approach using information of preceding bogies

\subsection{Track Trajectory}

To cope with the influences of the track trajectory the analytical model is extended by $a_{l a t}$ in the lateral direction and $M_{G}$ in the yaw motion. The required signals are directly feed into the models. Assuming that accurate values for $r_{T}$ and $\varphi_{T}$ are available from acceleration measurements in combination with track and satellite data, both values are regarded ideally. Hereby, a preview information is not relevant, because the signals fluctuate very slowly. To gain a value for $\varphi_{T}$, the rolling rate of the axle beam $\dot{\varphi}_{w}$ is obtained at the preceding axle beam. A low-pass filter is applied to suppresses the movement of the axle beam and attain a estimation for $\dot{\varphi}_{T}$. The preview signal at the trailing control is finalized with a time delay.

\subsection{Lateral Track Irregularities}

In reality, the lateral irregularity cannot be measured in a straight-forward way, but can be received from observers in future [3]. To evaluate the potential of the control nevertheless, $y_{T}$ is obtained ideally at the preceding running gear and pre-processed for the control of the trailing one. To deal with the rapid alteration of $y_{T}$ a desired lateral trajectory $y_{w}^{*}$ for the DIRW is specified on velocity and acceleration level. The aim of the control is, to center the wheel pair in the track and minimize flange contact. Therefore, the desired position has to be set to 
the center of the disturbed track: $y_{w}^{*}=0$. A low pass filter is applied to cut-off the highest dynamics of $y_{T}$, because the system cannot follow them due to the vehicle inertia. The signals are differentiated and time delayed to obtain $\dot{y}_{w}^{*}=\dot{y}_{T}^{\prime}$ and $\ddot{y}_{w}^{*}=\ddot{y}_{T}^{\prime}$.

\section{Results}

To validate the control approach a Simpack multibody model of a single wagon is set-up. In contrast to the synthesis model of the controller, the wagon is equipped with two running gears, each with a single wheel pair. Three optimization steps are performed for the integrated control. At first, the controller gains are optimized numerically on straight tracks to counterbalance the irregularities. Second, the cut-off frequency and the delay for $y_{T}^{\prime}$ are found and finally the parameters for $\dot{\varphi}_{T}^{\prime}$ on curved tracks are determined. The control torque is limited to $\Delta \tau=350 \mathrm{Nm}$. Therefore, a combined optimization criterion is used, to find a balance between lateral position and control effort.

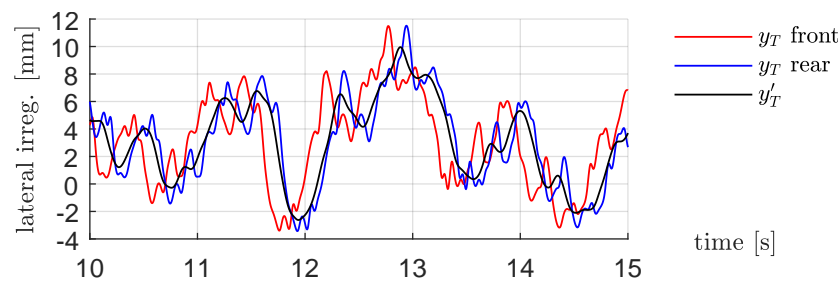

\begin{tabular}{|l|c|c|}
\hline Parameter & $y_{T}$ & $\dot{\varphi}_{T}$ \\
\hline$f_{\text {cut }}[\mathrm{Hz}]$ & 2.43 & 1.6 \\
\hline$\Delta t[\mathrm{~s}]$ & 0.039 & 0.024 \\
\hline
\end{tabular}

Fig. 4. Pre-processing of $y_{T}$ (left) with the parameters (right)

The optimized values for the pre-processing of the signals at a velocity of $v=300 \mathrm{~km} / \mathrm{h}$ are given in Fig. 4 on the right. As intended, the time delays are smaller than $\Delta t=0.168 \mathrm{~s}$, which is the gap that results from the wheel pair distance at the given velocity. The pre-processing for the lateral irregularity is displayed in Fig. 4 on the left. The irregularity $y_{T}$ at the front wheel pair (red) is filtered and time delayed (black). The desired trajectories $\dot{y}_{T}^{\prime}$ and $\ddot{y}_{T}^{\prime}$ for the rear control are obtained by derivation. It is obvious, that the desired trajectory occurs slightly before the irregularity at the rear wheel pair (blue) and that the high dynamics are filtered. Hence, superior positioning of the rear wheel pair concerning the low frequent disturbances of the track is enabled.

In case of the superelevation rate $\dot{\varphi}_{T}$ the low-pass filter with $f_{c u t}=1.6 \mathrm{~Hz}$ suppresses the rolling motion of the axle beam and the characteristic of the superelevation rate $\dot{\varphi}_{T}^{\prime}$ remains.

Finally, the simulation results with and without preview information $\left(\dot{y}_{w}^{*}, \ddot{y}_{w}^{*}=\right.$ $\left.0, a_{\text {lat }}=0, M_{G}=0\right)$ are compared. The scenario on a track with realistic irregularities includes all mentioned influences: a straight track is followed by a transition curve between $s=[0.6 \mathrm{~km}, 1.0 \mathrm{~km}]$ leading to an unbalanced curve 


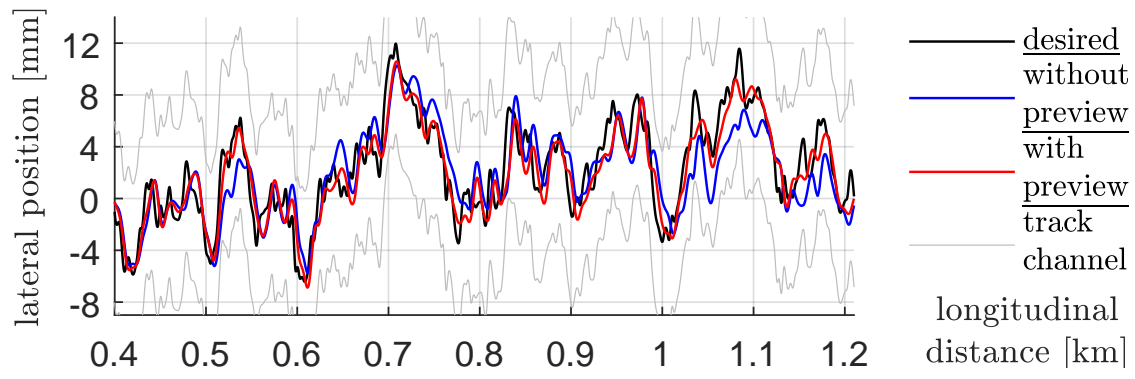

Fig. 5. Lateral position in track channel with and without preview information

with $r_{T}=7000 \mathrm{~m}, \varphi_{T}=0.07 \mathrm{rad}$ and respectively $a_{\text {lat }}=0.31 \mathrm{~m} / \mathrm{s}^{2}$. In Fig. 5 the lateral position of the rear wheel pair without (blue) and with preview information (red) is compared with the ideal position in the track center (black). The track channel (grey) displays the lateral clearance until the flange contact. In the straight track part, e.g. between $s=[0.5 \mathrm{~km}, 0.6 \mathrm{~km}]$, it is clearly visible that the position is improved due to the preview of $y_{T}^{\prime}$. From $s=0.6 \mathrm{~km}$ on, in the transition curve and during curving, the consideration of the track trajectory leads to an enhanced centering.

To comprise the whole approach over an extended distance of $s=1.7 \mathrm{~km}$, the root mean square values (rms) of the lateral displacement and the limited motor torque are compared in Table 2. The enhancement in the lateral position due to the integrated control to $62 \%$ of the displacement without additional information is significant. Remarkably, the control effort can be reduced simultaneously by 4.8 $\%$. Nevertheless, the approach is based on the availability of signals for the track characteristics. Therefore, the control is validated without information of $y_{T}$ as well $\left(\dot{y}_{w}^{*}, \ddot{y}_{w}^{*}=0 ; a_{\text {lat }}, M_{G}\right.$ included). The rms values show, that nevertheless a improved lateral positioning is attained and that the control effort stays low. An indicator for the wear in simulation is the $T_{\gamma}$ value, calculated as product of creep velocities and corresponding creep forces. Regarding the wear reduction with respect to a reference highspeed train with conventional wheelsets, the results of Kurzeck [10] are confirmed. With each of the proposed controllers only around $10 \%$ of the wear remains. Thereby, the consideration of preview information leads to a further decrease.

Table 2. Absolute mean wear $\left(T_{\gamma}\right)$ w.r.t reference vehicle and rms-values of control effort and lateral position

\begin{tabular}{|l|c|c|c|c|c|}
\hline Controller & $T_{\gamma}[\%]$ & $\Delta \tau_{\text {lim }}[\mathrm{Nm}]$ & $\Delta \tau_{\text {lim }}[\%]$ & $y_{w}[\mathrm{~mm}]$ & $y_{w}[\%]$ \\
\hline without preview & 11.4 & 182.0 & 100 & 2.19 & 100 \\
\hline with $\dot{y}_{T}^{\prime}, \ddot{y}_{T}^{\prime}, a_{\text {lat }}, M_{G}$ & 8.3 & 173.4 & 95.2 & 1.36 & 62.0 \\
\hline only $a_{\text {lat }}, M_{G}$ & 9.2 & 173.6 & 95.4 & 1.89 & 85.9 \\
\hline
\end{tabular}




\section{Conclusion And Outlook}

In this work the lateral guidance control design for a driven independently rotating wheel pair via feedback linearization is presented. It is based on an analytical model and guaranties the stability of the system. The control is extended to evaluate the potential of an integrated control structure using information of preceding bogies. The proposed enhanced control approach includes information arising from curving, transition curves and lateral irregularities. For this purpose, signals for the lateral irregularity and the alteration of the superelevation are obtained at a leading wheel pair and pre-processed for the controller of trailing wheel pairs. The control performance is optimized in multibody simulations and the results on a realistic track scenario reveal the potential of the preview information. The rms value of the lateral displacement can be reduced to $62 \%$ with a simultaneous decrease of the control effort of $4.8 \%$. Even without the preview of the track irregularities, only with information on the curving the lateral control is improved. Compared to a reference vehicle with conventional wheelsets, an enormous wear reduction by around $90 \%$ is attained. Hereby, the integrated control structure with preview information is advantageous and shows the lowest wear. Nevertheless, the concept is predicated on information of the track characteristics and the states of the running gear, which is partly not available in recent applications. Particularly, a measurement of $y_{T}$ is recently not implemented in reality, but the progress of the observer development for the states of the DIRW is promising [3]. Most certainly, the availability of track data will rise and more vehicle states can be observed for the realization of an enhanced control.

\section{References}

1. Farhat, N., Ward, C.P., Dixon, R., Goodall, R.M.: Benefits of mechatronically guided vehicles on railway track switches. Proceedings of the Institution of Mechanical Engineers, Part F: Journal of Rail and Rapid Transit (2018)

2. Grether, G.: Dynamics of a running gear with IRWs on curved tracks for a robust control development. PAMM 17(1), 797-798 (2017)

3. Heckmann, A., Keck, A., Schwarz, C.: Nonlinear observer design for guidance and traction of railway vehicles. In: 26th International Symposium on Dynamics of Vehicles on Roads and Tracks (2019). Accepted for publication

4. Heckmann, A., Lüdicke, D., Grether, G., Keck, A.: From scaled experiments of mechatronic guidance to multibody simulations of dlr's next generation train set. In: 25th International Symposium on Dynamics of Vehicles on Roads and Tracks, Dynamics of Vehicles on Roads and Tracks. CRC Press (2017)

5. Heckmann, A., Schwarz, C., Bünte, T., Keck, A., Brembeck, J.: Control development for the scaled experimental railway running gear of dlr. In: 24th Symposium of the International Association for Vehicle System Dynamics (IAVSD 2015) (2016)

6. Isidori, A.: Nonlinear control systems. Springer-Verlag, Berlin (1989)

7. Iwnicki, S. (ed.): Handbook of railway vehicle dynamics. Taylor \& Francis, Boca Raton (2006)

8. Joos, H.D., Bals, J., Looye, G., Schnepper, K., Varga, A.: A multi-objective optimisation-based software environment for control systems design. In: IEEE 
International Conference on Control Applications and International Symposium on Computer Aided Control Systems Design, pp. 7-14 (2002)

9. Kalker, J.J.: Three-dimensional elastic bodies in rolling contact. Solid mechanics and its applications. Kluwer Acad. Publ., Dordrecht [u.a.] (1990)

10. Kurzeck, B., Heckmann, A., Wesseler, C., Rapp, M.: Mechatronic track guidance on disturbed track: the trade-off between actuator performance and wheel wear. Vehicle System Dynamics 52(sup1), 109-124 (2014)

11. Thümmel, M., Looye, G., Kurze, M., Otter, M., Bals, J.: Nonlinear inverse models for control. In: 4th International Modelica Conference (2005) 